\title{
A Boolean algebra algorithm suitable for use in temperature-humidity control of a grafted seedling acclimatization chamber
}

\author{
Li-John Jou ${ }^{\text {a,b }}$, Chung-Min Liao ${ }^{\text {b,* }}$, Yi-Chich Chiu ${ }^{a}$ \\ a Department of Biomechatronic Engineering, National Ilan University, \\ Ilan, Taiwan 260, People's Republic of China \\ ${ }^{\mathrm{b}}$ Ecotoxicological Modeling Center, Department of Bioenvironmental Systems Engineering, \\ National Taiwan University, Taipei, Taiwan 10617, People's Republic of China
}

Received 1 July 2004; received in revised form 18 November 2004; accepted 31 December 2004

\begin{abstract}
The objective of this paper was to link a psychometric chart and Boolean algebra algorithms on deducing the procedural and logical issues of temperature-humidity environmental control for a grafted seedling acclimatization chamber. We developed a condition-sequence control circuit based on Boolean algebra algorithms to solve the complex logical problem existing in temperature-humidity environmental control procedures. A Matlab program was used to deduce and simplify the Boolean relations between input and output logical variables of the controller, whereas the correctness of Boolean function was verified by an Excel program. The Boolean functions were firstly transformed to switching (logical) circuits and subsequently transformed to a programmable logical controller (PLC) program systematically. We implemented the developed industrial wiring devices and PLC in designing a temperature-humidity environmental controller of an acclimatization chamber for grafted seedlings in that we adopted the nonlinear control technique associated with the conditionsequence control methodology as the main control scheme. On the other hand, we adopted the compound control action, two-position set point action, the time-delay control and the conditional action of free cooling as the main control operations for the controller. Our results demonstrate that the overall seasonal survival rate was $97 \%$ by using the Boolean algebra-based controlled
\end{abstract}

\footnotetext{
* Corresponding author. Tel.: +8862 2363 4512; fax: +886223626433.

E-mail address: cmliao@ntu.edu.tw (C.-M. Liao).
} 
chamber grafting, which was higher than that of $90 \%$ by employing the field grafted seedling operations.

(C) 2005 Elsevier B.V. All rights reserved.

Keywords: Acclimatization chamber; Condition-sequence control; Boolean algebra; Psychrometrics; Programmable logical controller

\section{Introduction}

Grafting can strengthen not only the disease resistance ability, but also can promote the production quality of vegetable/fruit crops. Grafted seedlings are extensively used in Asian countries such as Korea, Japan and Taiwan, where cultivated land is poor and intensively used. The purpose of grafting has greatly expanded because it reduces the effect of soil-borne infectious diseases, increases the ability to tolerate low-temperature and salt and wet-soil, enhances water and nutrient uptake and increase plant vigor to extend the duration of economical harvest time (Chiu et al., 1999). Grafted seedlings are popular in Taiwan for watermelon, bitter gourd, eggplant and cucumber, where watermelon is the majority with 30 million seedlings per year for about 20,000 ha of field (http://www.coa.gov.tw/statistic/newyearbook/PDF/).

Vegetable grafting joins the stock and scion together by a clipper and/or pipe. Since both the stock and scion are cut for grafting, they need to reconstruct their bundles in the process of acclimatization. For watermelon grafting, the stock is cut about 12 and 18-20 days after seeding in summer and winter, respectively. The scion merely needs 4 days to grow after seeding in summer and 7 days in winter. Generally, the seedlings take about 7-10 days to coalesce under controlled environment (Chiu et al., 1999). Both the grafting and post-grafting curing operations significantly affect the survival rate of grafted seedlings. Nobuoka et al. (1996) pointed out that higher relative humidity (RH) was advantageous to coalesce and to prevent the grafted seedlings of tomatoes from wilting. An improper curing environment will cause the seedlings to languish, produce over-growth, or even die.

In the present status of post-grafting curing operations, the seedlings are moved to the under protected cover in the field, where climate violently affects the curing environment. The farmers must regulate the indoor environment by venting, covering, shading, etc. when the weather changes. Chiu et al. (1999) suggested that the optimal air temperature, RH and light and dark interval period were $25-28{ }^{\circ} \mathrm{C}, 90 \%$ and $12 \mathrm{~h}$, respectively, for acclimating grafted seedlings of watermelon. Chang et al. (2003) suggested that the survival rate and quality of grafted seedlings were promoted if the $\mathrm{RH}$ in the acclimatization chamber was maintained at $80-90 \%$, whereas under $70 \% \mathrm{RH}$ both indexes were greatly decreased. An average light intensity of $25-49 \mu \mathrm{mol} \mathrm{m}^{-2} \mathrm{~s}^{-1}$ could promote the leaf area, and fresh and dry weight of grafted seedlings. The suitable nursing temperature would be at $26-29^{\circ} \mathrm{C}$, whereas at $32-35^{\circ} \mathrm{C}$, the leaf area, fresh and dry weight of grafted seedlings were significantly decreased.

Most mathematical models for microclimate control were mainly built on the theory of mass-energy equilibrium (Papadakis et al., 1994; Wang and Boulard, 2000; Young et 
al., 2000; Trigui et al., 2001; Pasgianos et al., 2003). In nonlinear conditions, the climate parameters in mathematical models are calculated by numerical methodologies such as Runge-Kutta and Newton-Raphson methods, which are complicated computational procedures. Most mathematical models were not easily implemented to control on-line, and the major difficulties were divergence problems caused by choosing wrong initial state-variable vectors.

Vassilyev (1997) suggested that it was better to consider the discrete time in logical methods for analysis and modeling of the controlled systems. Bemporad and Morari (1999) proposed a framework for modeling and controlling systems to describe interdependent physical laws, logic rules, and operating constrains, denoting as mixed logical dynamical (MLD) systems. MLD systems include linear hybrid systems, finite state machines, some classes of discrete event systems, constrained linear systems and nonlinear systems which can be approximated by piecewise linear functions. Boolean algebra plays a major role in computer sciences and can be used to represent sets and formulas of propositional logical and digital circuits (Buttner and Simonis, 1987). Boolean algebra algorithms are a conscientious deducing rule for deciding a logical control path. Rosen (1999) suggested that the Quien-McCluskey method could build optimal expressions by systematically grouping the together-products when the number of input logical variables exceeds four. The algorithm was tedious to use by hand, yet was very suitable to program for computer calculation (Ross and Wright, 1999). Thus, we adopted the Quien-McCluskey method as the main simplifying methodology for the present logical networks.

The objective of this paper was to develop an environmental controller for providing a suitable curing environment in a grafted seedlings acclimatization chamber. Specifically, the main goals of this paper were 4-fold: (1) to construct a logical control mathematical methodology by applying Boolean algebra algorithms to solve the complex logical problem existing in temperature-humidity environmental control procedures, (2) to deduce and simplify the Boolean expressions between input and output variables for the logical system by using the Matlab program, using an Excel program to verify the correctness of Boolean function, (3) to transform Boolean functions to switching (logical) circuits and then to progressively transform to a PLC program and (4) to implement the Boolean algebra algorithm in designing a temperature-humidity environmental controller of an acclimatization chamber for grafted seedlings in that the nonlinear control technique associated with the condition-sequence control methodology was adopted as the main control scheme. The compound control actions, two-position set point action, the time-delay control and the conditional action of free cooling were adopted as the main control operations for the controller.

\section{Materials and methods}

\subsection{Experiment}

The dimensions of the acclimatization chamber were $540 \mathrm{~cm} \times 265 \mathrm{~cm} \times 210 \mathrm{~cm}$ with a $5 \mathrm{~cm}$ thick insulation and $185 \mathrm{~cm}$ inner height (Fig. 1). The chamber accommodated nine 
(a)

(b)
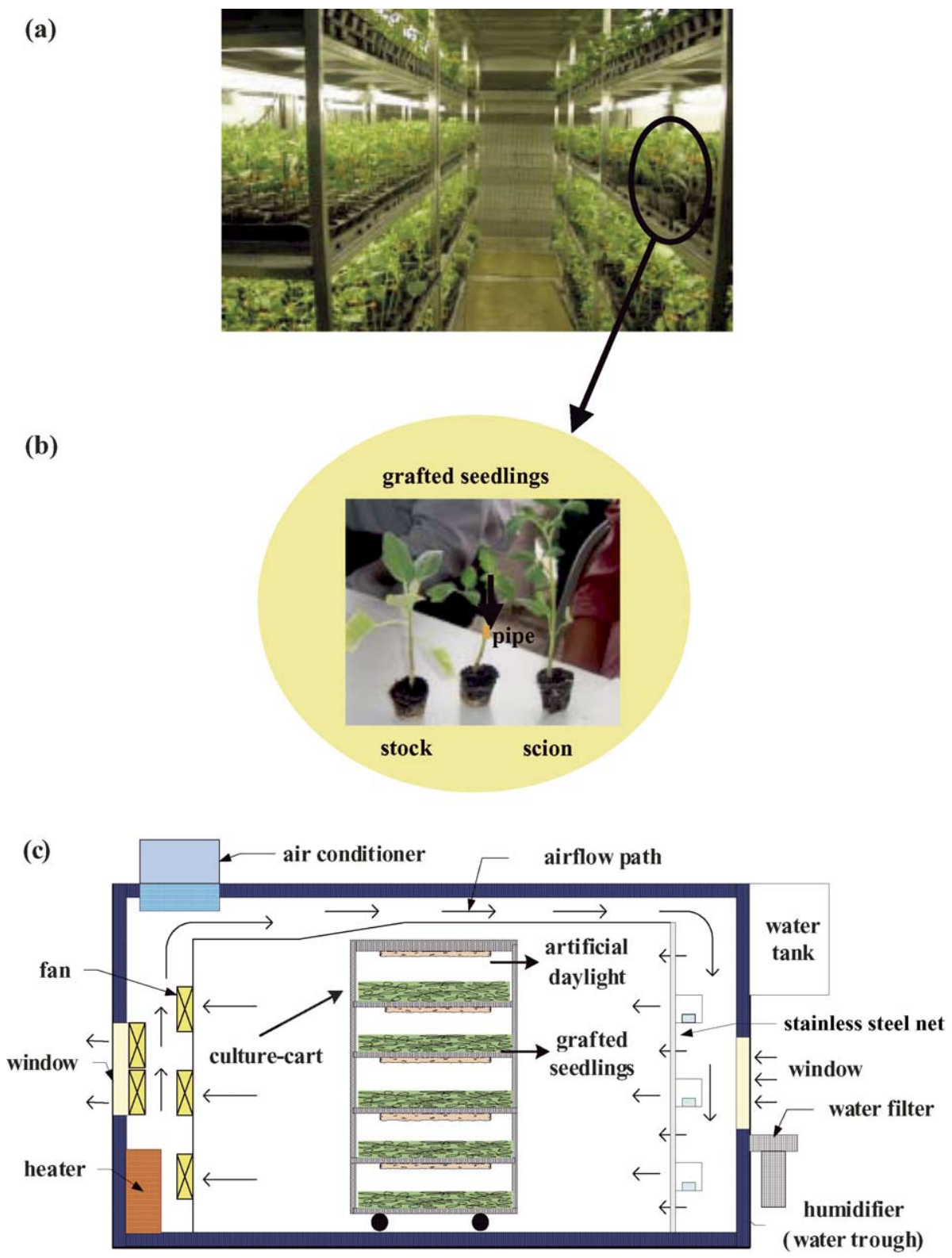

Fig. 1. (a) The seedlings were kept on the culture-carts in the chamber after grafting, (b) vegetable grafting is to join the stock and scion together by a clipper and/or pipe and (c) cross section of an acclimatization chamber for grafted seedlings. 
culture-carts. Each cart consisted of five layers with four trays in each layer. The chamber had a capacity of 12,960 seedlings and 72-cell trays were used. Chiu et al. (1999) suggested that an air speed lower than $0.2 \mathrm{~m} \mathrm{~s}^{-1}$ could prevent the grafted seedlings from wilting and achieve the reconstruction of bundles. The uniform air circulation and distribution in the chamber was regulated by three rows of $10-\mathrm{cm}$ fans with five fans for each row, and a stainless steel net.

The chamber equipped with artificial daylight, free cooling, humidifier, heater and air conditioner was used to provide a suitable curing environment for seedling after grafting (Fig. 1c). A $40 \mathrm{~W}$ straight plant-lamp (FL-40SBR/38) was mounted on the top of each layer in the cart to maintain constant spatial illumination in the chamber. The plant-lamps in the chamber were controlled by a 24 -h timer. The chamber can be regarded as a heatisolated system from outside so that sunlight does not affect the inner environment. The heat produced by plant-lamps was followed by the assigned temperature-humidity control strategies. Ventilation openings installed on both sides of the chamber were controlled by timer to periodically accommodate $\mathrm{CO}_{2}$ concentration. If the outside temperature and $\mathrm{RH}$ were suitable for curing of grafted seedlings, ambient air was introduced to regulate the chamber environment to save energy. The artificial daylight and $\mathrm{CO}_{2}$ concentration in the chamber were then regulated by the time-sequence control method. We used ultrasonic humidifiers with the total capacity of $9 \mathrm{Lh}^{-1}$ to humidify the incoming air to prevent ulceration of the coalescent part of the grafted seedlings from fog. A $2 \mathrm{KW}$ heater was used to warm or dehumidify indoor environment. An air conditioner $\left(20.0 \mathrm{MJ} \mathrm{h}^{-1}\right)$ was equipped to provide a cooler environment.

Chiu et al. (1999) pointed out that the spatial variation of temperature, RH and air speed in the chamber was insignificant in that variations for temperature, $\mathrm{RH}$ and air speed were around $1{ }^{\circ} \mathrm{C}, 3.6 \%$ and $<0.2 \mathrm{~m} \mathrm{~s}^{-1}$, respectively. As a result, the microclimate in the chamber can be treated as a completely mixed and uniform environment. The variation of temperature and humidity then can be simplified to a function of only time. The temperature-humidity environment was modulated by a control method of a feedback conditions-sequence.

The acclimatization chamber was equipped with an environmental controller deployed by linking Boolean functions and a psychrometric chart to regulate indoor environment. Timers controlled hourly air exchange to modulate $\mathrm{CO}_{2}$ concentration. We verified the functions of the environmental controller seasonally. Temperature-humidity recorders (HOBO-H08032-08, Onset Computer Corp., U.S.A.) were installed both inside and outside of the chamber for recording the temperature-humidity variations on a daily basis at a 1-min sampling rate.

We processed the acclimating experiments of the grafted seedlings to obtain the survival rates for comparing the grafted seedlings kept in the chamber with that moved in the field under protected cover during the acclimating stage in spring (autumn), summer and winter. Consequently, the survival rates of the grafted seedlings were qualified and quantified by measuring the physical survival indices of grafted seedlings including the length of stock and scion, the diameter of stock and scion, leaf area and chlorophyll as a consulting index of evaluation. We observed the state of reconstructing bundles in the coalescent part by fluorescence in order to determine whether the grafted seedlings were sustained-healthily survived. Each obtained datum was 1000 replicate plants after 14 days of grafting in spring, summer and winter, respectively. 


\subsection{Control framework}

Fig. 2a illustrates the control framework for the system. The target of temperaturehumidity environmental field confined by a psychrometric chart was regarded as a finite area bounded by lower and upper limits of temperature $\left(T_{\mathrm{L}}-T_{\mathrm{H}}\right)$ and $\mathrm{RH}\left(\mathrm{RH}_{\mathrm{L}}-\mathrm{RH}_{\mathrm{H}}\right)$, respectively. According to the upper-lower state of temperature and $\mathrm{RH}$ sensors, the temperature-humidity environment was mainly divided into nine parts ( $\square-i$ ) in a psychrometric chart (Fig. 2b and c). There were 162 states of temperature-humidity environment associated with the outside, inside and illuminative time conditions. When the initial or proceeding state was disturbed by external factors such as weather changes and worker activities, and temperatures and RH fell outside the control target area, the control strategies were quickly actuated to achieve the control target by operating mechanical equipment. Free cooling was operated automatically to save energy while indoor and outdoor temperature-humidity were both in the control target. The control paths formulated by Boolean functions and psychrometric processes could systematically perform and achieve the control rules and target settings.

The inputs of temperature and $\mathrm{RH}$ sensor states for PLC were the discrete finite states. In most control circuits of complex electrical systems, it is frequently necessary to make intricate interconnections of relay contacts and switches. We adopted Boolean algebra algorithms to deduce the switching function that completely expressed as whole control processes in any state. A temperature-humidity environmental controller in a grafted seedlings acclimatization chamber was implemented by adding on-line control strategies of timesequence. The combination control circuit for the system mainly consisted of forward (comparing and two-position control actions), core (logic- and time-sequence) and outputload circuits (Fig. 3).

\subsection{Psychrometric processes}

Fig. 2b shows a psychrometric chart of cooling, dehumidifying, heating and humidifying control processes in a grafted seedlings acclimatization chamber. The area confined by lines was a control target of temperature and humidity in a psychrometric chart, whereas the area bounded by dotted lines was the control target of two-position setting. In summer, the outdoor temperature was higher than that of indoor and the initial state of the temperature-humidity environment in the chamber was assumed on the outside boundary $(\square-c)$. The psychrometric processes formulated by the principles of air-conditioning and humidifying are shown as paths $1-4$ in Fig. 2 b.

Since the outdoor temperature is typically lower than that of indoors in winter, the indoor $\mathrm{RH}$ is usually higher than the upper-limit $\mathrm{RH}\left(\mathrm{RH}_{\mathrm{H}}\right)$. For this condition, the heater is started to reduce the indoor $\mathrm{RH}$ until the two-position setting of $\mathrm{RH}\left(\mathrm{RH}_{\mathrm{H}^{\prime}}\right)$ is reached. The control process is shown as path 5 in Fig. $2 \mathrm{~b}$. When the indoor temperature-humidity environment is in the g- or h-state, the heater and humidifiers are operated by formulating the control paths to regulate the indoor temperature-humidity environment for achieving the target of two-position setting (paths 6-10 in Fig. 2b).

Fig. 2c shows a psychrometric chart of natural heating, dehumidifying, humidifying and free cooling control processes in an acclimatization chamber for grafted seedlings. 


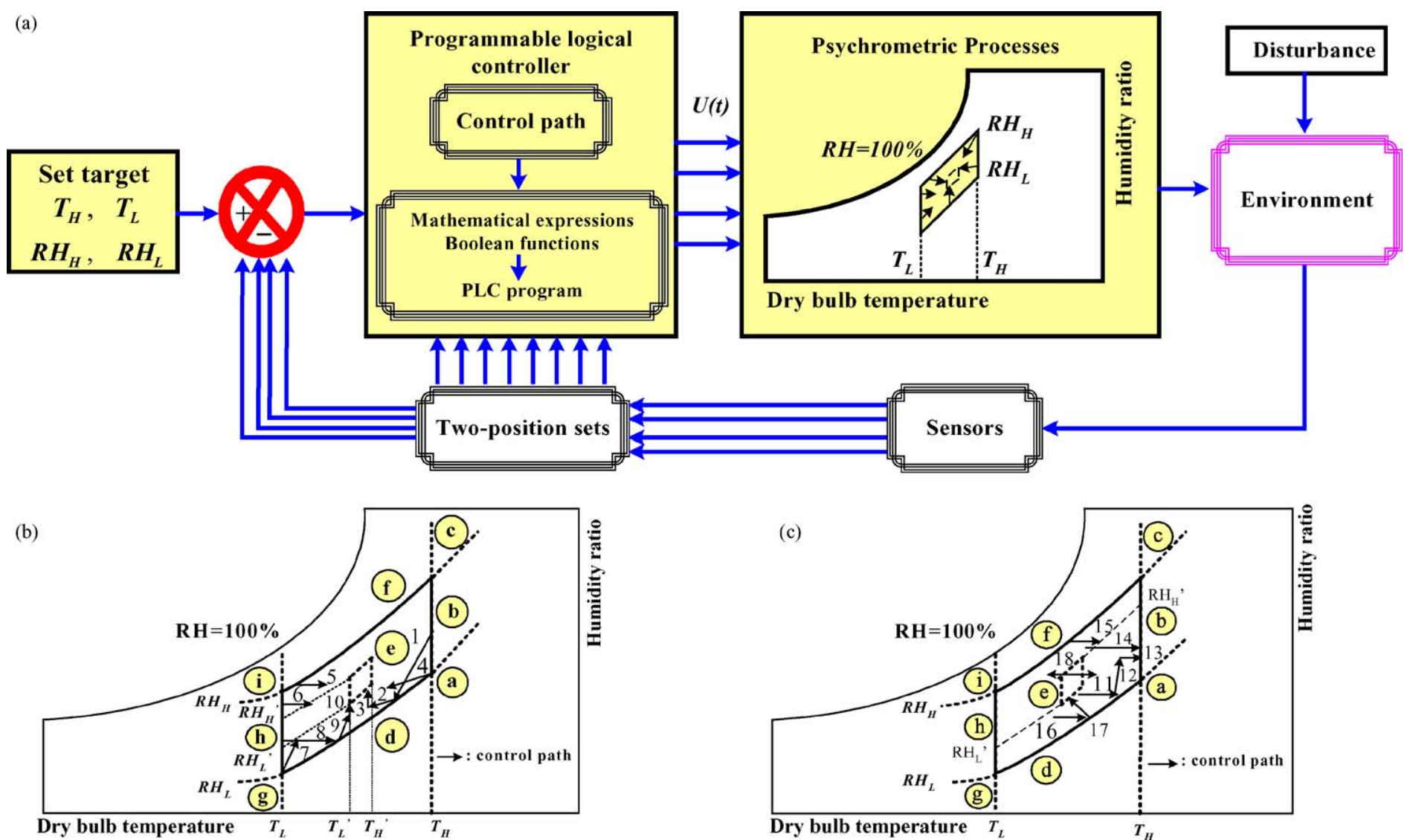

Fig. 2. (a) The diagram of a control framework for the system in a grafted seedlings acclimatization chamber, (b) a psychrometric chart of cooling, dehumidifying, heating and humidifying control processes in a grafted seedlings acclimatization chamber and (c) a psychrometric chart of natural heating, dehumidifying, humidifying and free cooling control processes in an acclimatization chamber for grafted seedlings. 
Forward circuit

(comparing \& two-position control action)

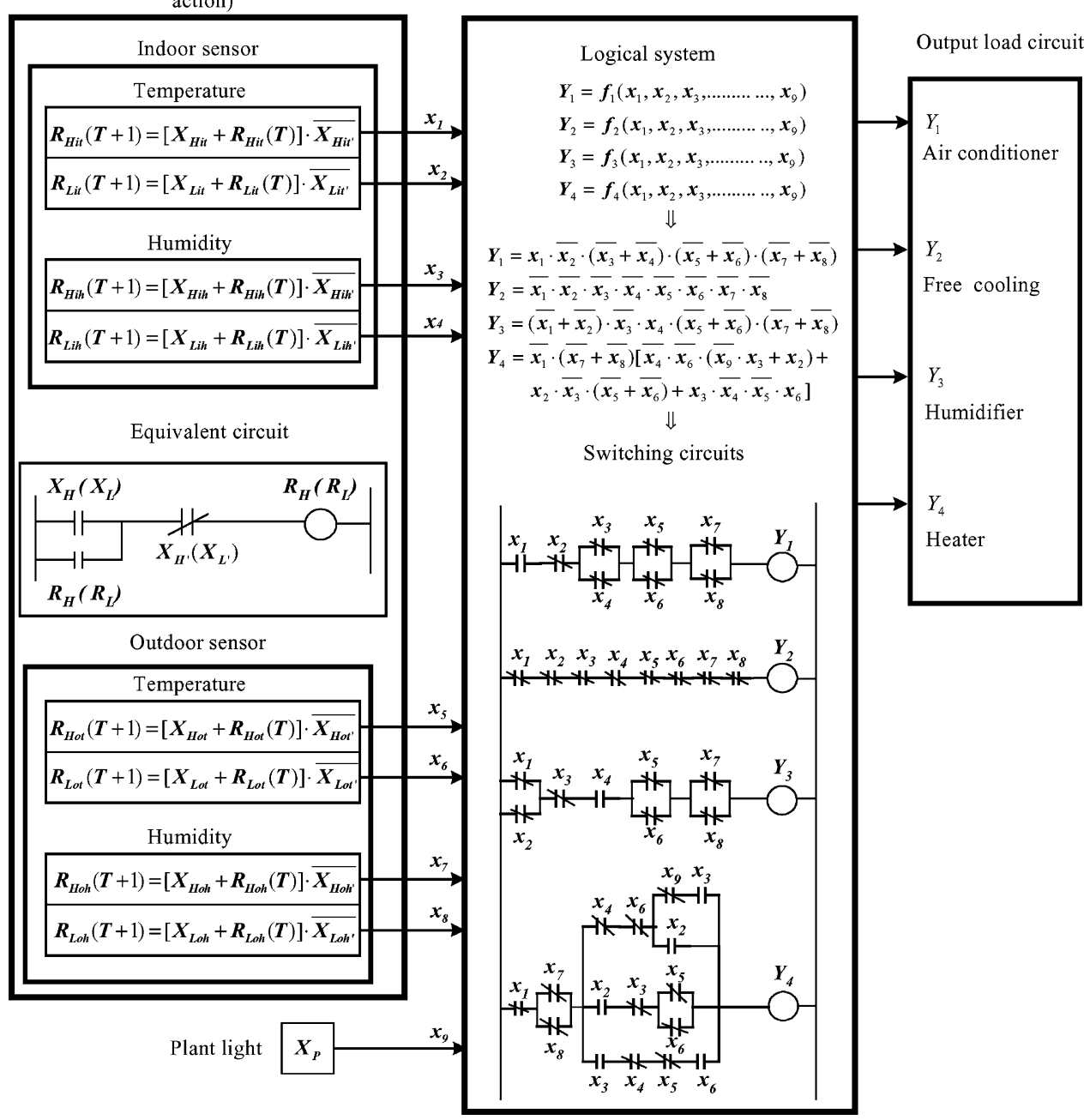

Fig. 3. The combinative diagram of the temperature-humidity environmental controller for a grafted seedlings acclimatization chamber.

In summer, when the heat sources in the chamber were produced by the plant-lamps, the psychrometric processes expressed by the paths of natural heating, dehumidifying and humidifying are as shown by paths 11-14 in Fig. 2c. If the indoor temperature-humidity environment is in the f-state, the heat sources produced by the plant-lamps can be used to decrease the indoor $\mathrm{RH}$ without operating the heater. The control process is shown as path 15 in Fig. 2c. When the plant-lamps were switched on, whereas the indoor temperature-humidity environment was still kept at the f-state after exceeding $3 \mathrm{~min}$. The heater could be started to reduce the indoor RH automatically. 
In spring, autumn, and summer nights, when the indoor temperature-humidity environment was in the d- or e-state, the indoor temperature-humidity environment was periodically regulated by the free cooling. The plant-lamps were then switched on by a timer. If the RH in the chamber was reduced by the heat, resulting in a RH that was lower than the lowerlimit $\mathrm{RH}\left(\mathrm{RH}_{\mathrm{L}}\right)$, the humidifiers must be switched on until the $\mathrm{RH}$ reached the target of two-position setting (paths 16-18 in Fig. 2c).

\subsection{Mathematic model}

A control circuit consisting of forward, core and output-load circuits was integrated to implement the system (Fig. 3). Both two-position setting and comparing circuits were major parts of the forward circuit. The comparing and two-position control action circuits could be represented as a mathematical pattern of equivalence by the following expressions:

$$
\begin{aligned}
& X_{\mathrm{L}}=\left\{\begin{array}{ll}
1 & X(t) \leq w \\
0 & X(t)>w
\end{array}, \quad X_{\mathrm{L}^{\prime}}=\left\{\begin{array}{ll}
1 & X(t) \geq z \\
0 & X(t)<z
\end{array},\right.\right. \\
& R_{\mathrm{L}}(T+1)=\left[X_{\mathrm{L}}+R_{\mathrm{L}}(T)\right] \cdot \bar{X}_{\mathrm{L}^{\prime}}, \\
& X_{\mathrm{H}}=\left\{\begin{array}{ll}
1 & X(t) \geq u \\
0 & X(t)<u
\end{array}, \quad X_{\mathrm{H}^{\prime}}=\left\{\begin{array}{ll}
1 & X(t) \leq v \\
0 & X(t)>v
\end{array},\right.\right. \\
& R_{\mathrm{H}}(T+1)=\left[X_{\mathrm{H}}+R_{\mathrm{H}}(T)\right] \cdot \bar{X}_{\mathrm{H}^{\prime}}, \\
& {[w, z] \quad \text { and }[v, u] \in R,}
\end{aligned}
$$

where $X(t)$ is a time-dependent state-variable produced by a sensor, $X_{\mathrm{H}}$ and $X_{\mathrm{H}^{\prime}}$ the uncomplemented logical variables of the upper-limit contact commanded by comparing circuits, $X_{\mathrm{L}}$ and $X_{\mathrm{L}^{\prime}}$ the uncomplemented logical variables of the lower-limit contact commanded by comparing circuits, $\bar{X}_{\mathrm{H}^{\prime}}$ and $\bar{X}_{\mathrm{L}^{\prime}}$ the complemented logical variables, $R_{\mathrm{H}}(T)$ and $R_{\mathrm{L}}(T)$ the logical variables denoted by virtual relays at the present state $(T), R_{\mathrm{H}}(T+1)$ and $R_{\mathrm{L}}(T+1)$ the logical variables denoted by virtual relays at the next state $(T+1)$ and $w, z, v$ and $u$ are the setting values in registers and comparators.

The core circuit mainly consisted of logical and time-sequence circuits. They were also expressed as a mathematical pattern of equivalence by the Boolean functions. The $n$ input variables canonical form (the sum of products) of Boolean functions can be expressed as:

$$
\begin{aligned}
Y_{k}= & f_{k}\left(x_{1}, x_{2}, \ldots x_{n}\right)=\left(c_{k 1} \overline{x_{1}} \cdot \overline{x_{2}} \cdot \overline{x_{3}} \ldots \overline{x_{n-1} \cdot x_{n}}+c_{k 2} \overline{x_{1}} \cdot \overline{x_{2}} \cdot \overline{x_{3}} \ldots \overline{x_{n-1}} \cdot x_{n}\right. \\
& +c_{k 3} \overline{x_{1}} \cdot \overline{x_{2}} \cdot \overline{x_{3}} \ldots \cdot x_{n-1} \cdot \overline{x_{n}}+\ldots c_{k 2^{(n-1)}} x_{1} \cdot x_{2} \cdot x_{3} \ldots x_{n-1} \cdot \bar{x}_{n} \\
& \left.+c_{k 2^{n}} x_{1} \cdot x_{2} \cdot x_{3} \ldots x_{n-1} \cdot x_{n}\right)=\sum_{i=1}^{2^{n}} c_{k i} \cdot m_{i-1}
\end{aligned}
$$


where $k$ is the number of output variables, $x$ the uncomplemented logical variables, $\bar{x}$ the complemented logical variables, $C_{k i}$ the constant ( 1 or 0$)$ and $m_{i-1}$ is the numerical form expressed by the product of logical variables.

We used a Boolean matrix form to express the Boolean functions (Edwards, 1972):

$$
[C] \cdot X]=F],
$$

where $\left.[C]=\left[C_{k i}\right]_{k \times 2^{n}}, X\right]=\left[x_{1}, x_{2}, \ldots, x_{n}\right]^{\mathrm{T}}$, and $\left.F\right]=\left[f_{1}, f_{2}, \ldots, f_{k}\right]^{\mathrm{T}}$ in that $[C]$ is the constant $(0$ or 1$)$ matrix in that $[C]$ is also the matrix established and transposed by the truth table of output operations, $X]$ the input logical variable vector and $F]$ is the output logical function vector.

In this paper, the number of input logical variables $X$ produced by a virtual relay is $9(n=9)$ and the number of output logical function $f$ is $4(k=4)$. Logical issues linked by a temperature-humidity environmental control procedure and a psychrometric chart associated with a Boolean algebra algorithm were hidden in the truth table including $2^{9}=512$ states. We adopted a Boolean matrix form to systematically express the functions (the detailed derivation may be requested from the authors). The constructed processes and results are described in the subsequent sections.

\subsection{Deducing Boolean functions and implementation}

We treated $w$ and $z$ as well as $u$ and $v$ (Eqs. (1) and (2)) representing the two-position setting value of the lower and upper limit of temperature and $\mathrm{RH}$, respectively, in that we defined $w=T_{\mathrm{L}}\left(\mathrm{RH}_{\mathrm{L}}\right), z=T_{\mathrm{L}^{\prime}}\left(R H_{\mathrm{L}^{\prime}}\right), u=T_{\mathrm{H}}\left(\mathrm{RH}_{\mathrm{H}}\right)$ and $v=T_{\mathrm{H}^{\prime}}\left(\mathrm{RH}_{\mathrm{H}^{\prime}}\right)$. In the forward circuits (Fig. 3), we defined the variables $X_{\mathrm{Hit}}, X_{\mathrm{Lit}}, X_{\mathrm{Hih}}, X_{\mathrm{Lih}}, X_{\mathrm{Hot}}, X_{\mathrm{Lot}}, X_{\mathrm{Hoh}}, X_{\mathrm{Loh}}, X_{\mathrm{Hit}}$, $R_{\text {Lit }}, R_{\text {Hih }}, R_{\text {Lih }}, R_{\text {Hot }}, R_{\text {Lot }}, R_{\text {Hoh }}$ and $R_{\text {Loh }}$ corresponding to the denoting subscripts of H, $\mathrm{L}, \mathrm{i}, \mathrm{o}, \mathrm{t}, \mathrm{h}$ and ', respectively, representing the upper limit, the lower limit, indoor, outdoor, temperature, humidity and two-position setting, e.g.,

$$
\begin{aligned}
& X_{\mathrm{Hit}}=\left\{\begin{array}{ll}
1 & X_{\mathrm{it}}(t) \geq T_{\mathrm{H}} \\
0 & X_{\mathrm{it}}(t)<T_{\mathrm{H}}
\end{array}, \quad X_{\mathrm{Hit}^{\prime}}=\left\{\begin{array}{ll}
1 & X_{\mathrm{it}}(t) \leq T_{\mathrm{H}^{\prime}} \\
0 & X_{\mathrm{it}}(t)>T_{\mathrm{H}^{\prime}}
\end{array},\right.\right. \\
& R_{\mathrm{Hit}}(T+1)=\left[X_{\mathrm{Hit}}+R_{\mathrm{Hit}}(T)\right] \cdot \bar{X}_{\mathrm{Hit}^{\prime}}, \\
& X_{\mathrm{Lit}}=\left\{\begin{array}{ll}
1 & X_{\mathrm{it}}(t) \leq T_{\mathrm{L}} \\
0 & X_{\mathrm{it}}(t)>T_{\mathrm{L}}
\end{array}, \quad X_{\mathrm{Lit}^{\prime}}=\left\{\begin{array}{ll}
1 & X_{\mathrm{it}}(t) \leq T_{\mathrm{L}^{\prime}} \\
0 & X_{\mathrm{it}}(t)>T_{\mathrm{L}^{\prime}}
\end{array},\right.\right. \\
& R_{\mathrm{Lit}}(T+1)=\left[X_{\mathrm{Lit}}+R_{\mathrm{Lit}}(T)\right] \cdot \bar{X}_{\mathrm{Lit}^{\prime}}
\end{aligned}
$$

The other expressions of forward circuits could also be obtained (Fig. 3). The input and output variables $\left(x_{1}, x_{2}, \ldots, x_{9}\right.$ and $\left.Y_{1}, \ldots, Y_{4}\right)$ for the core circuit were respectively expressed as the following expressions:

$$
\begin{aligned}
& x_{1}=R_{\mathrm{Hit}}(T+1), x_{2}=R_{\mathrm{Lit}}(T+1), x_{3}=R_{\mathrm{Hih}}(T+1), x_{4}=R_{\mathrm{Lih}}(T+1), \\
& x_{5}=R_{\mathrm{Hot}}(T+1), x_{6}=R_{\mathrm{Lot}}(T+1), x_{7}=R_{\mathrm{Hoh}}(T+1) \text { and } x_{8}=R_{\mathrm{Loh}}(T+1) .
\end{aligned}
$$


According to the definition of the input variables, we can deduce the Boolean functions in the core circuit. Firstly, we established the truth table of input states in terms of the state of temperature-humidity sensors. The truth table of output operations was formulated to construct psychrometric processes. The Boolean expressions between input and output variables for the logical system were deduced and simplified by using the Matlab program developed by the Quien-McCluskey algorithm, whereas an Excel program was also used to verify the correctness of Boolean functions (the detailed derivation may be requested from the authors). The simplified Boolean expression of nine input variables and four output variable is:

$$
\begin{aligned}
& Y_{1}=x_{1} \cdot \overline{x_{2}} \cdot\left(\overline{x_{3}}+\overline{x_{4}}\right) \cdot\left(\overline{x_{5}}+\overline{x_{6}}\right) \cdot\left(\overline{x_{7}}+\overline{x_{8}}\right) \\
& Y_{2}=\overline{x_{1}} \cdot \overline{x_{2}} \cdot \overline{x_{3}} \cdot \overline{x_{4}} \cdot \overline{x_{5}} \cdot \overline{x_{6}} \cdot \overline{x_{7}} \cdot \overline{x_{8}} \\
& Y_{3}=\left(\overline{x_{1}}+\overline{x_{2}}\right) \cdot \overline{x_{3}} \cdot x_{4} \cdot\left(\overline{x_{5}}+\overline{x_{6}}\right) \cdot\left(\overline{x_{7}}+\overline{x_{8}}\right) \\
& Y_{4}=\overline{x_{1}} \cdot\left(\overline{x_{7}}+\overline{x_{8}}\right) \cdot\left[\overline{x_{4}} \cdot \overline{x_{6}} \cdot\left(\overline{x_{9}} \cdot x_{3}+x_{2}\right)+x_{2} \cdot \overline{x_{3}} \cdot\left(\overline{x_{5}}+\overline{x_{6}}\right)+x_{3} \cdot \overline{x_{4}} \cdot \overline{x_{5}} \cdot x_{6}\right]
\end{aligned}
$$

We secondly transformed Boolean functions into switching circuits (Fig. 3) and then the transformation of a PLC program was systematically obtained.

We transformed the equivalent mathematical pattern of forward circuits and Eqs. (5) and (6) into a PLC program (Fig. A.1a in Appendix A). Similarly, the other equations as shown in Fig. 3 were also completely transformed into the PLC program. The switching circuits of core (Fig. 3) in conjunction with the time-sequence control functions were then used to protect the operational equipment and to promote the modulating performance. By using these equivalent mathematical forms for control switching circuits, we can rapidly and progressively transform the switching circuits of core (Fig. 3) into a PLC program (Fig. A.1b in Appendix A). The completed PLC program could be implemented to control the temperature-humidity environment of a grafted seedling acclimatization chamber (the detail of the transformative procedures and results may be requested from the authors).

\section{Results and discussion}

Based on the meteorological information of the Ilan region of Taiwan during 1971-2000 (http://www.cwb.gov.tw/v4e/index.htm), the lowest average temperatures occurring in January and February were 16.0 and $16.4{ }^{\circ} \mathrm{C}$, respectively, whereas the highest average temperatures occurring in July and August were 28.4 and $28.0^{\circ} \mathrm{C}$, respectively. The average temperatures in other months were $17.3-26.5^{\circ} \mathrm{C}$. We, therefore, chose the experimental time interval in winter, spring and summer, respectively, from January 20 to February 10 (temperatures were $\left.10-18{ }^{\circ} \mathrm{C}\right)$, March 20 to April $10\left(20-25^{\circ} \mathrm{C}\right)$ and July 20 to August 10 $\left(25-38^{\circ} \mathrm{C}\right)$. 
We interpreted the control results associated with the response variation curves of temperature and RH on a daily basis during winter, spring and summer. Moreover, a psychrometric chart of the control target area confined by setting temperatures and RHs was also used to illustrate the controller performance. We compared the grafted seedlings acclimatized under the cover in field with acclimating in the chamber, based on the survival rate of grafted seedlings in different seasons and acclimatization environments.

The set points for temperature ranges of free cooling in winter, spring and summer were 10-18, 18-26 and $26-34{ }^{\circ} \mathrm{C}$, respectively, whereas the set points for $\mathrm{RH}$ ranges of free cooling were 50-80, 70-90 and 80-90\%, respectively, in sunny, cloudy and rainy days. The control target range of the temperature-humidity environment in the chamber was set at $26-28{ }^{\circ} \mathrm{C}$ and $80-90 \%$ in any season.

Fig. 4 illustrates the results when the chamber was expectably controlled at a specific set point ranges of $26-28{ }^{\circ} \mathrm{C}$ temperature and $80-90 \% \mathrm{RH}$. The time period of illumination in the chamber was from 19:00 to 07:00. The heat sources produced by the plant-lamps $(1800 \mathrm{~W})$ were used to keep the indoor temperature at $27.0-27.9^{\circ} \mathrm{C}$ without engaging
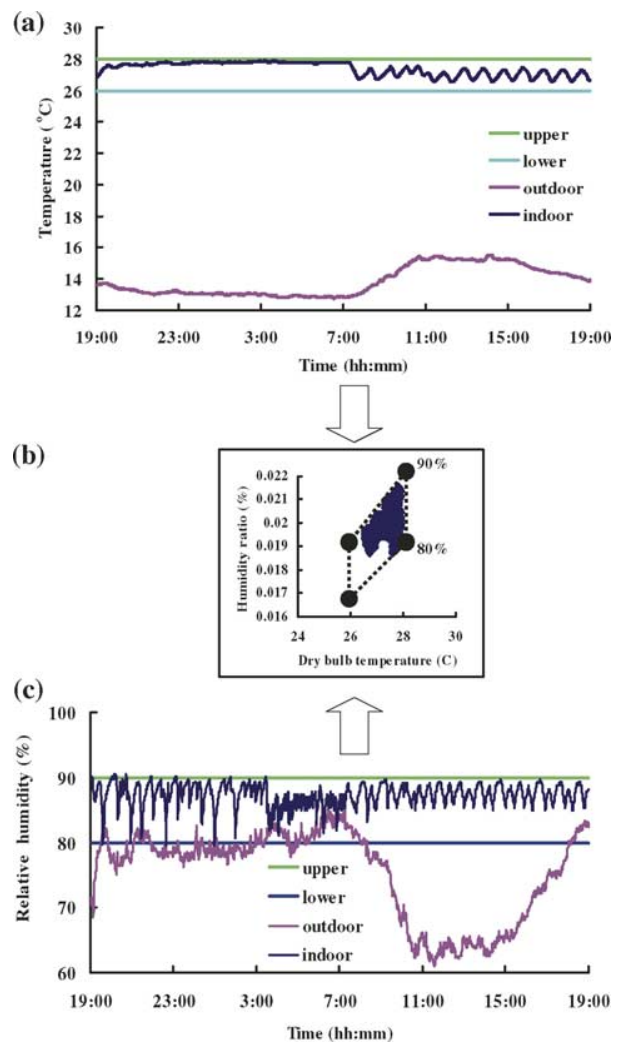

Fig. 4. The performance of the temperature-humidity environmental controller in an acclimatization chamber for grafted seedlings during winter: (a) response variation curves of temperature, (b) a psychrometric chart illustration of control target area for both temperature and $\mathrm{RH}$ and (c) response variation curves of $\mathrm{RH}$. 
the heater. The environmental operations of free cooling were intermittently performed to decrease the inner surplus heat, yet to change the indoor RH at 80.1-89.9\%. The humidifiers were intermittently operated to increase the indoor RH. The plant-lamps were switched off by a timer after 07:00 h. Since the outdoor temperature was lower than that of indoor in winter, the indoor RH was usually higher than the upper-limit $\mathrm{RH}$ of $90 \%$. The heater was started to reduce the indoor $\mathrm{RH}$ until the two-position setting of $\mathrm{RH}$ at $85 \%$. When the heater was intermittently used to warm and dehumidify the indoor temperature-humidity environment, the chamber was operated by a closed system. The indoor temperature and $\mathrm{RH}$ were regulated at $26.8-27.3^{\circ} \mathrm{C}$ and $86.2-88.8 \%$, respectively.

The control target of the indoor temperature-humidity environment in spring is shown in Fig. 5. The daily indoor temperature and $\mathrm{RH}$ were controlled at $26.2-27.9^{\circ} \mathrm{C}$ and $84.6-87.9 \%$, respectively. The time period of without illumination in the chamber was from 19:00 to 07:00. Since the plant-lamps $(1200 \mathrm{~W})$ were switched off after 19:00 h, the heat in the chamber was not enough to keep the indoor lower-limit temperature at $26^{\circ} \mathrm{C}$. From 17:40 to 21:50, the free cooling was operated to regulate the indoor environment until

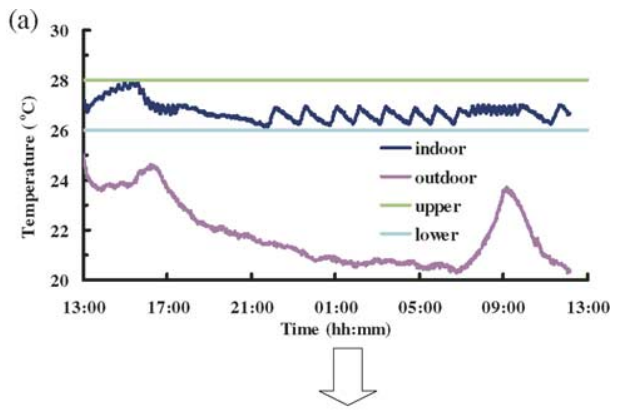

(b)
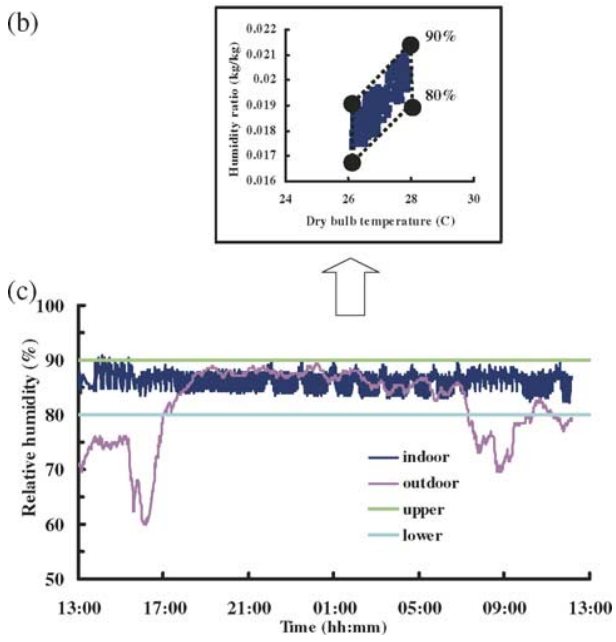

Fig. 5. The performance of the temperature-humidity environmental controller in an acclimatization chamber for grafted seedlings during spring: (a) response variation curves of temperature, (b) a psychrometric chart illustration of control target area for both temperature and $\mathrm{RH}$ and (c) response variation curves of RH. 
the indoor temperature was lower than the lower-limit temperature of $26^{\circ} \mathrm{C}$. The heater was immediately and intermittently started to warm the indoor temperature until the plant-lamps $(1200 \mathrm{~W})$ were started at 07:00 $\mathrm{h}$ by a timer. The free cooling was automatically performed to modulate the indoor environment again after 07:00 $\mathrm{h}$.

Fig. 6 illustrates the indoor temperature and $\mathrm{RH}$ environments were regulated at $27.3-27.6^{\circ} \mathrm{C}$ and $83.1-87.2 \%$, respectively, during summer. When the initial temperature and $\mathrm{RH}$ in the chamber were $29.1^{\circ} \mathrm{C}$ and $79.5 \%$, the air conditioner associated with the humidifiers was automatically operated to regulate the indoor temperature and $\mathrm{RH}$ until the indoor temperature was lower than the upper-limit temperature of $28^{\circ} \mathrm{C}$. The plant-lamps $(1200 \mathrm{~W})$ were switched on after 19:00 h, the free cooling associated with the humidifiers was firstly performed to decrease the indoor surplus heat and to keep the indoor temperature and $\mathrm{RH}$ at $27.3-27.6^{\circ} \mathrm{C}$ and $83.1-87.2 \%$, respectively. Since the time period of without illumination in the chamber was regulated from 07:00 to 19:00 h, if the outdoor temperature and $\mathrm{RH}$ were suitable for modulating the indoor temperature and $\mathrm{RH}$ of the control

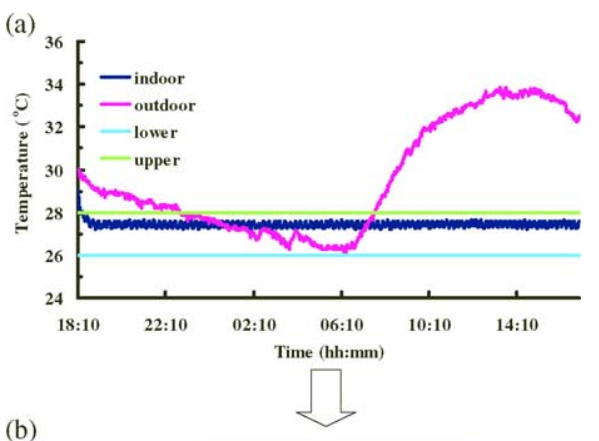

(1)
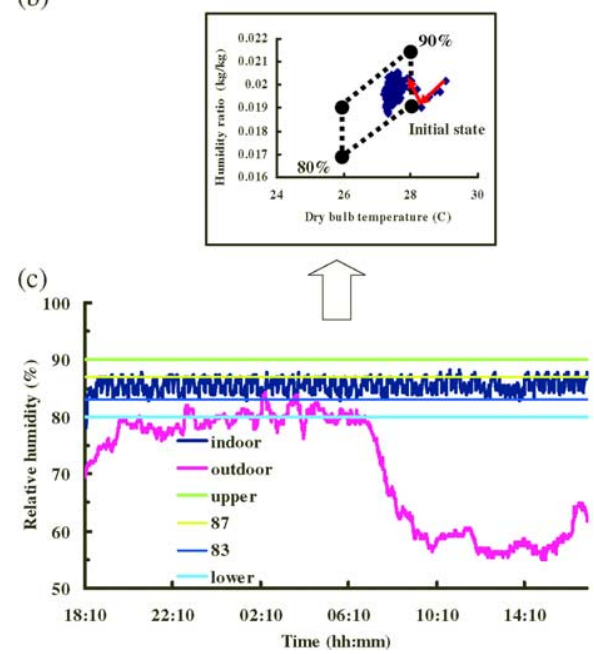

Fig. 6. The performance of the temperature-humidity environmental controller in an acclimatization chamber for grafted seedlings during summer: (a) response variation curves of temperature, (b) a psychrometric chart illustration of control target area for both temperature and RH and (c) response variation curves of RH. 


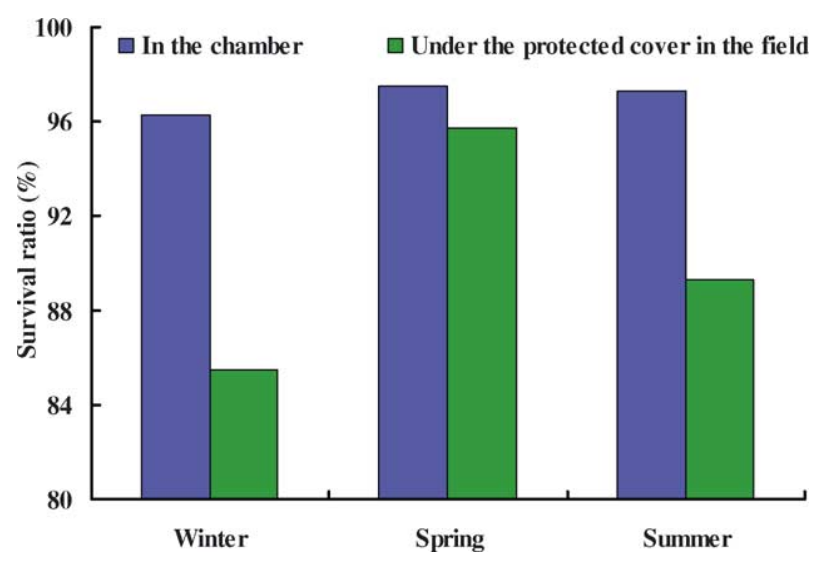

Fig. 7. The survival ratios (\%) of grafted seedlings in different seasons and acclimatization environments.

target, the free cooling associated with the humidifiers was firstly engaged. Otherwise, the controller would close the vents to form a closed system, and the other suitable operations were performed in accordance with indoor states and psychrometric processes settings.

The acclimating experiments of the seedlings were, respectively, kept in the chamber and moved to the field under protected cover after grafting. Fig. 7 indicates that the survival rate of grafted seedlings kept in the chamber were 96.2, 97.5 and $97.4 \%$ in spring, summer and winter, respectively. An overall seasonal survival rate was $97.03 \pm 0.64 \%$ (mean \pm S.D.) by using the Boolean algebra-based controlled chamber grafting, that is higher than that of $90.17 \pm 5.16 \%$ when employing field operations. A higher survival rate is shown for the seedlings acclimated in the chamber after grafting as compared to those grafted seedlings acclimated under the protected cover in the field, especially in winter (Fig. 7). A major complication in the evaluation of the survival rates for grafted seedling acclimated in the chamber and under the protected cover exists because of the uncertainty from the environmental management variability (especially in the field) and variance among individuals caused by the grafting procedure.

After acclimating, we can adjust the indoor temperature-humidity environment of the control target to adapt the grafted seedlings to the seasonal changes for increasing the survival ratio of the domesticate period. The domesticate period of the grafted seedlings acclimated in the chamber were reduced from 21 to 14 day during winter comparing with the conventional culture in the under protected cover in the field.

A dynamic heat balance differential equation in the chamber was obtained to estimate and demonstrate the indoor temperature difference rate caused by the controlled heater during winter (e.g., $\mathrm{d} T_{\mathrm{r}} / \mathrm{d} t=0.084{ }^{\circ} \mathrm{C} \mathrm{min}^{-1}$ ). Similarly, the indoor temperature and moisture variation rate caused by air conditioner and humidifiers were also obtained during summer and spring by calculating the equation of mass-energy equilibrium and processing experiment, respectively. In this paper, we considered not only the traditional models such as the differential or dynamic equations in the form of "input-output" as $y=C x$, where $x$ is input, $y$ the output and $C$ is presented by some operational mechanisms. We adopted logical models having also a type "input-output," e.g., the formula $\forall x(A(x)) \Rightarrow \exists y B(x, y)$, where $A$ and $B$ 
are formula-mechanisms of a logical language, that can be interpreted as a statement that for every value of the input $x$ such that $A(x)$ is valid and the output has the value $y$ such that $B(x, y)$ holds (Vassilyev, 1997). For example, the blocks marked "F-212W(WNDW)" and "F-12W(CMP)" (Fig. A.1a in Appendix A) take the data from the sensors and convert them into a form which the Boolean logical controller can employ, i.e., the data from a set of temperature and humidity sensors might be converted from measurable and quantifiable degrees into Boolean logical state variables. The output of the logic controller is actually a set or range of response (e.g., a heater and humidifiers, or only a heater with a proportional action). Hence, a system behavior is described through the discrete definition or properties of values of variables, e.g., Eqs. (5) and (6) $\Rightarrow \forall x(A(x))$, where $A$ is the functions combined with the discrete states and two-position actions, and Eqs. (7)-(10) $\Rightarrow y(B(x, y))$, where $B$ is the logical mechanisms combined with the Boolean algebra and psychrometric processes. Therefore, input and output are connected indirectly by these properties corresponding to each other and being described in a logical function.

A logical control system applies a set of control rules suitable for various situations which may overlap or confine each other. The final operation of action is a judgment, which is some appropriate combination of all relevant factors. A traditional feedback control system makes its decision about what to do based on either a mathematical model of the process or a proportional-integral-derivative (PID) controller (a fixed set of mathematical relationships). In the present research, the logical controller is an implement based on the control paths (e.g., logical rule and operating constrains) coupling with mass-energy equilibrium equations and experiences. It was simpler to solve the complex problems existing in the mixed system that includes some classes of discrete event systems, constrained linear systems and nonlinear systems which can be approximated by piecewise linearization than that of the classical control. In future work, Boolean logical controllers can be used in conjunction with traditional PID controllers or fuzzy logic controllers more precisely and completely to account for the real systems.

\section{Conclusions}

Our work demonstrates that automatic networks (switching circuits) linked and formulated by both Boolean functions and psychrometric processes are the important components of the environmental controller for a grafting seedling acclimatization chamber. It is very useful and suitable for use in proposing some qualitative method of their control target instead of exhaustive numerical methodology under all admissible initial states, parameters, and disturbances. The mathematical models described by logical variables were easily implemented to control on-line by a PLC or a graphic control program (e.g., LabVIEW) in a PC. The methodology could overcome the difficulties of accidentally occurring divergent problems caused by choosing initial state-variable vectors.

Using the methodology and technique, we implemented the environmental controller for grafted seedlings in the acclimatization chamber. The controller performances demonstrate that the methodology is realizable and the control system can satisfactorily achieve expected goals for providing suitable curing conditions and modulating the environmental factors such as temperature, $\mathrm{RH}$, illumination and $\mathrm{CO}_{2}$ for grafted seedlings in the acclima- 
tization chamber. The acclimating experiments of the grafted seedlings resulted in an overall seasonal survival rate of $97.03 \pm 0.64 \%$ by using Boolean algebra-based chamber grafting, which was higher than that of $90.17 \pm 5.16 \%$ by employing field operations.

\section{Acknowledgements}

This work was supported by the National Science Council of Republic of China under Grant NSC91-2313-B197-007. Sincere thanks go to seedlings farm owners for providing valuable information and for use of their seedlings farms, without which this research work would have not been possible. We also thank Dr. Suming Chen, editor Dr. Sidney Cox and two anonymous reviewers for providing constructive comments in the manuscript.

\section{Appendix A}

Fig. A.1.

(a)
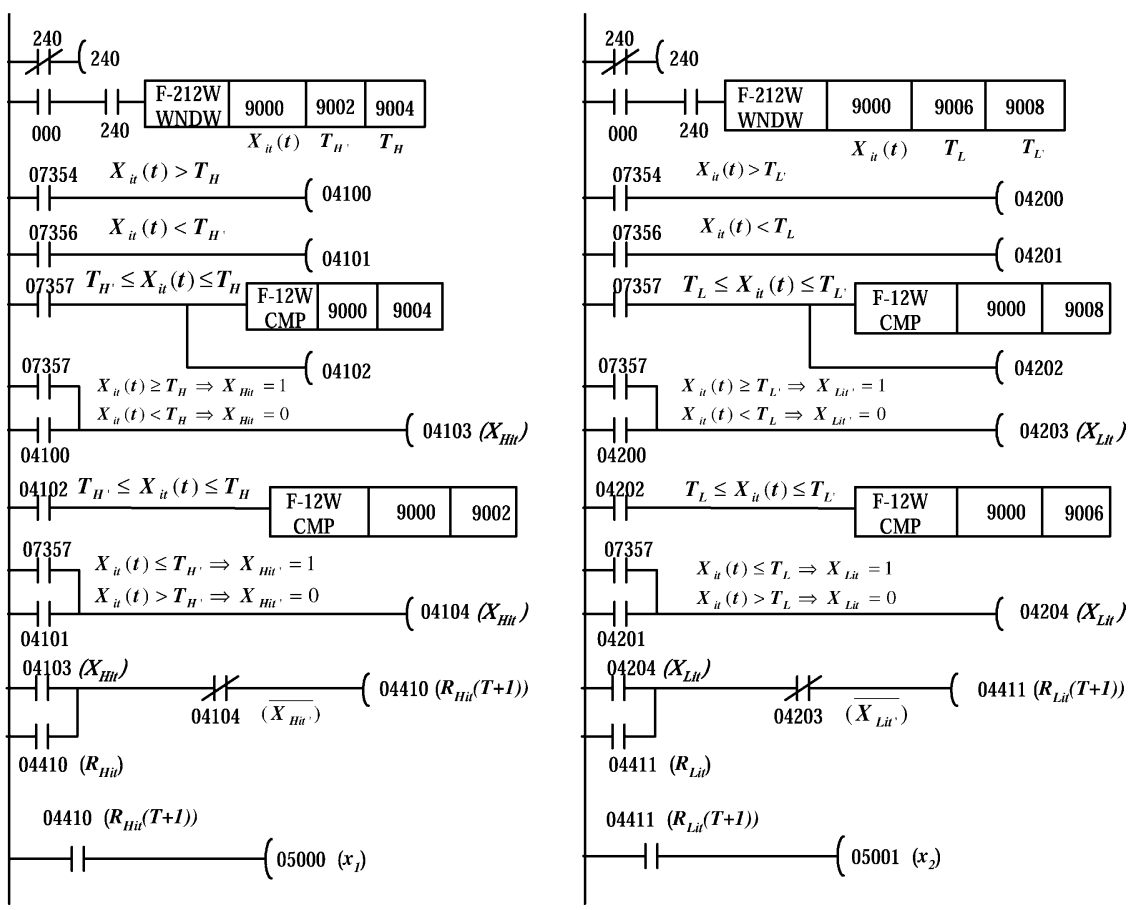

Fig. A.1. (a) The equivalent mathematical pattern of forward circuits as well as Eqs. (5) and (6) were transformed into a PLC program and (b) a PLC program was developed by transforming and compiling the switching circuits of core. 


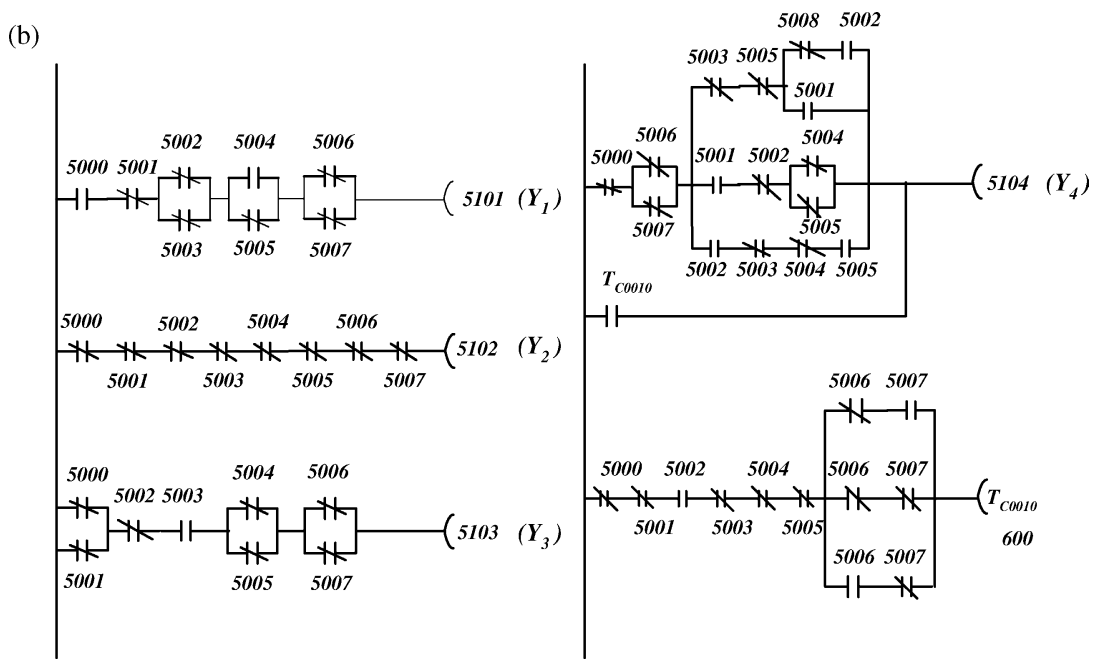

Fig. A.1. (Continued).

\section{References}

Bemporad, A., Morari, M., 1999. Control of systems integrating logic, dynamics, and constraints. Automatica 35, 407-427.

Buttner, W., Simonis, H., 1987. Embedding Boolean expressions into logic programming. J. Symbolic Comput. 4, 191-205.

Chiu, Y.C., Jou, L.J., Chen, S., 1999. Development on an acclimatization chamber for grafted seedlings. ASAE Paper No. 99-5014, ASAE, St. Joseph, MI.

Chang, Y.C., Chiu, Y.C., Chen, S., 2003. The study of acclimatization environmental condition on grafted seedlings of 'Empire No.2' watermelon. J. Chin. Soc. Hort. Sci. 49, 275-288.

Edwards, C.R., 1972. The logic of Boolean Matrices. J. Comput. 15, 247-253.

Nobuoka, T., Oda, K., Sasaki, H., 1996. Effect of relative humidity, light intensity and leaf temperature of tomato. J. Jpn. Soc. Hort. Sci. 64, 859-865.

Papadakis, G., Frangoudakis, A., Kyritsis, S., 1994. Experimental investigation and modelling of heat and mass transfer between a tomato crop and the greenhouse environment. J. Agric. Eng. Res. 57, 217-227.

Pasgianos, G.D., Arvanitis, K.G., Polycarpou, P., Sigrimis, N., 2003. A nonlinear feedback technique for greenhouse environmental control. Comput. Electron. Agric. 40, 153-177.

Rosen, K.H., 1999. Discrete Mathematics and its Applications, fourth ed. WCB/McGraw-Hill Co., Singapore, pp. 593-617.

Ross, K.A., Wright, C.R.B., 1999. Discrete Mathematics, fourth ed. Prentice Hall Inc., New Jersey, pp. 517-524.

Trigui, M., Barrington, S., Gauthier, L., 2001a. A strategy for greenhouse climate control. Part I. Model development. J. Agric. Eng. Res. 78, 407-413.

Trigui, M., Barrington, S., Gauthier, L., 2001b. A strategy for greenhouse climate control. Part II. Model validation. J. Agric. Eng. Res. 79, 99-105.

Vassilyev, S.N., 1997. Logical approach to control theory and application. Nonlin. Anal. Theor. Meth. Appl. 30, 1927-1937.

Wang, S., Boulard, T., 2000. Predicting the microclimate in a naturally ventilated plastic house in a mediterranean climate. J. Agric. Eng. Res. 75, 27-38.

Young, P., Price, L., Berckmans, D., Janssens, K., 2000. Recent developments in the modeling of imperfectly mixed airspaces. Comput. Electron. Agric. 26, 239-254. 